\title{
Effect of targeted nursing applied to SLE patients
}

\author{
XIANGYING ZHANG $^{1}$, YALI TIAN ${ }^{2},{\text { JUNBAO } \mathrm{LI}^{3} \text { and XINGLI ZHAO }}^{4}$ \\ Departments of ${ }^{1}$ Rheumatism and Immunology, ${ }^{2}$ Pediatric Surgery, ${ }^{3}$ Emergency and \\ ${ }^{4}$ Oncology, Binzhou People's Hospital, Binzhou, Shandong 256600, P.R. China
}

Received February 10, 2016; Accepted March 18, 2016

DOI: 10.3892/etm.2016.3173

\begin{abstract}
The aim of the present study was to evaluate the efficacy of targeted nursing for patients with systemic lupus erythematosus (SLE). A total of 114 patients clinically diagnosed with stable SLE were prospectively selected. The patients were randomly divided into the regular special nursing group, comprising 56 patients and the targeted nursing group (i.e., taylor made according to different pathogenic conditions and treatment period), comprising 58 patients. The patients received standard medical treatment for SLE, irrespective of their group, and the efficacy of targeted nursing on disease activity, incidence of complications, therapeutic compliance, quality of life and nursing satisfaction was compared with regular special nursing. The patients were followed up for a period of 20 months. The results showed that, disease activity and injury index score and incidence of complications were significantly less in the targeted nursing group than in the regular special nursing group $(\mathrm{P}<0.05)$. Additionally, therapeutic compliance, quality of life score and nursing content satisfaction were significantly higher in the targeted nursing group in comparison with the regular special nursing group $(\mathrm{P}<0.05)$. Thus, the results indicated that targeted nursing significantly improved therapeutic compliance and quality of life, and simultaneously, reduced complications and disease activity in patients receiving standard treatment for SLE.
\end{abstract}

\section{Introduction}

The incidence of systemic lupus erythematosus (SLE) in China is $\sim 50-60 / 100$ thousand and is higher in women than men (1). SLE is an autoimmune disease with the features of inflammation involving multiple systems of the body, chronic progression and repeated attack. Currently, no established standard treatment method is available, and the preferred treatment is the long-term administration of glucocorticoid or a combination

Correspondence to: Junbao Li, Department of Emergency, Binzhou People's Hospital, 511 Yellow River Seven Road, Binzhou, Shandong 256600, P.R. China

E-mail: 1i_junbao1@163.com

Key words: targeted nursing, systemic lupus erythematosus, quality of life, autoimmune disease of multiple immune inhibitors (2). The severe complications associated with SLE including, lupus nephrosis, lupus encephalopathy, grave pneumonitis and heart failure, have a damaging effect on the body, mind and members of the family of patients, especially women during the reproductive period (3).

Besides correct treatment, scientific health propagation, and professional and human nursing patterns are important factors affecting prognosis of the patients (4). Since regular special nursing only observes the basic principles of nursing and does not involve the different extents and treatment period of the disease, it needs further improvement (5). Targeted nursing is based on the 'society-psychology-biology' medical pattern, showing the 'integrated, high-quality, continuous' nursing concept, and achieves a positive effect in multiple fields (6).

Thus, we carried out the present study to evaluate the efficacy of targeted nursing over regular special nursing in SLE patients who presented to the Department of Rheumatism and Immunology.

\section{Materials and methods}

Patients. A total of 114 patients who were diagnosed with stable SLE between May 2013 and May 2015 were selected prospectively. The diagnosis of SLE was established in accordance with the diagnosis and classification standard revised by the American Rheumatism Academy in 1997 (7). The inclusion criteria for the study were: i) Age $\geq 18$ and $<70$ years; ii) first diagnosed and treated; and iii) have good cognition and compliance. The exclusion criteria were: i) Severe complications, severe pathogenic condition with bad predicted prognosis at activity period; ii) inability to endure therapeutic drug or appearance of allergy leading to termination of the treatment, voluntarily or inadequate clinical data; and iii) co-existence of other diseases, such as primary glomerulonephritis, liver or renal function injury or blood clotting mechanism disorder.

The present study was approved by the independent ethics committee of Binzhou People's Hospital (Shandong, China). Informed consent from patients and their family members was obtained prior to inclusion of the patients in the study.

By using the patients' registration number (descending order) of the Binzhou People's Hospital, patients bearing an odd number were grouped in the regular nursing group (56 patients) and patients bearing an even number were grouped into the targeted nursing group (58 patients). The regular nursing group comprised 50 females and 6 males, with an average age of $42.5 \pm 13.6$ years. The duration of the disease 
varied from 1 month to 1 year with an average of 3.5 months and the average education time was $13.6 \pm 2.5$ years. The targeted nursing group comprised 51 females and 7 males with an average age of $43.2 \pm 12.5$ years. The duration of disease ranged from 1.5 months to 1.5 years with an average of 3.7 months and an average education time of $13.5 \pm 2.3$ years.

Regular and targeted nursing. All the patients, irrespective of their groups, received standard clinical treatment for SLE and only the nursing differed between the groups. The components of regular specific nursing included: i) Dissemination of information on disease features, complications, possible outcome, importance of medical therapy and its possible complications and outcomes, management of sickrooms, patient attendance, necessary psychological interview and dredge; ii) nursing after multiple invasive operations including periodical exchange of detained needle, prompt tube sealing, checking loosening; iii) solving the problems of patients in time and reporting to physicians in charge; and iv) prompt execution of medical order.

Three phases of targeted nursing. The primary phase, which induced the release period, consisted of establishing basic intervention, acquiring high trust, understanding the detailed pathogenic condition, psychological reaction, social roles, family, economy and cognitive extent of the disease. This phase involved providing related journal abstracts, health pamphlets by professional nurses, discussing basic knowledge, including nature, diagnosis, treatment methods and prognosis of SLE assisted by definitions, figures and related cases, thereby informing the patients that SLE is a lifetime disease with complex and fickle pathogenesis.

Targeted nursing also included the application of varying methods to different individuals to conduct psychological dredge. The methods employed included interventions such as understanding, comforting, assisting, encouraging and supporting patients, to stimulate active psychological factors of the patients and provide them hope, thereby strengthening self-nursing. The nurses were also required to supervise medication during hospitalization and guide family members to assist in supervising medication by regularizing medication time, applying reminders such as using an electronic clock, medication diary and cell phones, and establish a record which would be convenient for follow-up. The patients were also informed of adverse reactions of the drugs, and equipped to prevent and deal with such reactions in due course. The patients were educated to prevent and deal with related complications, including herpes zoster, wound surface infection (wet therapy use, selection of debridement gel and silver ion dressings, seepage absorption in order to attend to the wound surface, and combined antibiotics to prevent infection), malnutrition (patients with a poor digestive function, were required to increase appetite and consume an increased amount of food with high protein, high fiber and low lipids that was easy to digest, as well as soft food, and consumption of multiple small meals), mouth ulcer, toxic epidermal necrolysis, report examined results within an allocated period of time, inform doctors whether there were abnormal hemogram, blood clotting function, liver and kidney function or cardiac muscle, and administer relevant treatment.
The secondary phase, involved nursing instructions on discharge from the hospital. For instance, a self-made SLE patient leaving hospital instruction plan was provided, distributed by professional nurses from the Department of Rheumatism and Immunology and explained in sequence. The content of the plan included medicines, daily health care knowledge, infection prevention, proper diet, inducement avoidance, contraception at unstable pathogenic condition, importance of consistent treatment, and special clinical service appointment.

The third phase, which involved continuous nursing following discharge from hospital, included establishing confidence, improving quality of life, regular treatment and reassessment, and health enhancement. In addition, a follow-up group was established and information was recorded. There were also regular follow ups in various forms including by telephone, MSN, QQ and email, and guiding medication and regular follow-up by clinical service.

Assessment of disease activity. The disease activity was assessed using SLEDAI rating scales in which the integral score was: 0-4, no activity; 5-9, slight activity; 10-14, midrange activity; and $>15$, severe activity.

Assessment of injury index score. The injury index score was assessed using SLICC/ACR and the integral method was used to assess the extent of injury of 12 organs, in which an index of 12-41 indicated worse prognosis.

Assessment of treatment compliance. The Likert scaling method was utilized to evaluate the compliance of medical treatment in which there were 10 items on the inquiry form. A score of 0 indicated 'No compliance'; 1, 'partial compliance'; 2 , 'complete compliance', and a full score was 20. Complete compliance was defined as active medication to the medical order, inactive medication according to medical order with irregular drug consumption was defined as partial compliance; and refusal to take the drug according to medical order as well as drug withdrawal or change of the dosage as the individual was defined as no compliance.

Assessment of quality of life. The SF-36 rating scale was used which contained 36 items and 8 dimensions. The primary score in each dimension was standardized into a final score, and the final score was between 0 and 100, with the higher score indicating improved quality of life.

Statistical analysis. SPSS 19.0 software was used to analyze the data. Quantitative results were presented as median \pm standard deviation, and the numbers were expressed as a median or percentage. The t-test and $\chi^{2}$ test were used for comparison of the results between groups. $\mathrm{P}<0.05$ was considered to indicate a statistically significant difference.

\section{Results}

Disease activity, injury index score and incidence of complications. The gender, age, pathogenesis and education time of the patients between the regular special nursing and targeted nursing groups had no significant difference $(P>0.05)$. The disease activity, injury index score and the incidence of 
Table I. Disease activity, injury index score and incidence of complications.

Incidence of complications

\begin{tabular}{|c|c|c|c|c|c|c|c|c|c|}
\hline Group & $\begin{array}{c}\text { Patients, } \\
\text { n }\end{array}$ & $\begin{array}{c}\text { Disease } \\
\text { activity } \\
\text { score }\end{array}$ & $\begin{array}{l}\text { Injury } \\
\text { index } \\
\text { score }\end{array}$ & $\begin{array}{c}\text { Infection, } \\
\mathrm{n}\end{array}$ & $\underset{n}{\text { Malnutrition, }}$ & $\begin{array}{c}\text { Lupus } \\
\text { encephalopathy, } \mathrm{n}\end{array}$ & $\begin{array}{c}\text { Lupus } \\
\text { nephrosis, n }\end{array}$ & $\begin{array}{c}\text { Others, } \\
n\end{array}$ & $\begin{array}{c}\text { Total } \\
\text { incidence } \\
\mathrm{n}(\%)\end{array}$ \\
\hline $\begin{array}{l}\text { Regular special } \\
\text { nursing }\end{array}$ & 56 & $8.4 \pm 1.5$ & $27.2 \pm 4.6$ & 5 & 4 & 2 & 2 & 1 & $14(25.0)$ \\
\hline Targeted nursing & 58 & $6.2 \pm 1.3$ & $18.5 \pm 4.2$ & 2 & 2 & 1 & 1 & 0 & $6(10.3)$ \\
\hline $\mathrm{t}\left(\chi^{2}\right)$ & & 5.624 & 5.768 & & & & & & 4.230 \\
\hline P-value & & 0.026 & 0.023 & & & & & & 0.040 \\
\hline
\end{tabular}

Table II. Therapeutic compliance, quality of life and nursing satisfaction.

\begin{tabular}{lccc}
\hline Group & Compliance score & Life quality score & Nursing satisfaction score \\
\hline Regular special nursing & $7.7 \pm 1.6$ & $72.4 \pm 12.6$ & $75.6 \pm 14.4$ \\
Targeted nursing & $15.6 \pm 2.2$ & $86.9 \pm 15.5$ & $88.7 \pm 17.5$ \\
T & 5.239 & 5.524 & 5.957 \\
P-value & 0.033 & 0.026 & 0.018 \\
\hline
\end{tabular}

complications were significantly decreased in the targeted nursing group than that of the regular special nursing group $(\mathrm{P}<0.05$, Table I).

Therapeutic compliance, life quality score and nursing satisfaction. The patients in the targeted nursing group showed significantly high therapeutic compliance, life quality score and nursing satisfaction in comparison with patients of the regular special nursing group $(\mathrm{P}<0.05$, Table II).

\section{Discussion}

In the present study, we investigated the efficacy of targeted nursing in patients with SLE. The results showed that, disease activity and injury index score and incidence of complications were significantly decreased in the targeted nursing group compared to that of the regular nursing group. Furthermore, targeted nursing significantly improved therapeutic compliance, quality of life and nursing content satisfaction compared to regular special nursing. Targeted nursing management is needed for clinical work and nursing operation, in addition to important interventions to improve nursing quality and achieve a harmonious physician-patient relationship. Targeted nursing emphasizes specialization and individuation of nursing, and fully utilizes nursing resources, carrying out specific nursing services to different patients, which yields a positive effect in the treatment of SLE (8).

The compliance of hormone therapy is the key to stabilization of SLE pathogenic condition and to control recurrence. The lack of relevant knowledge and supervision of medication, psychological fear, obvious adverse reaction, chronic pathogenesis, lack of economic resources and social support are all important factors affecting treatment compliance (9). Due to lack of supervision from health care workers following discharge of patients from the hospital, unwillingness to continue medication after disappearance of symptoms, not turning up for reassessment, reduced consumption of prescribed drugs or number of times, and changing drugs or administering dosage other than that prescribed by the medical staff lead to poor prognosis and even disease recurrence. Targeted nursing is patient-oriented and aimed at improving the quality of life of the patients during hospitalization and after discharge (10). It improves the nursing by combining oral and written propagation, in the form of lectures, bulletin boards, informal discussions between nurses and patients, and other individuation methods through nursing intervention for patients with complications according to their actual conditions.

The quality of life is defined by WHO as 'the experience of living conditions related to their goals, expected values, standard and attention point for people in different cultural and value systems' (11). Targeted nursing requires nursing workers to show human concern and integrate nursing concept for the patients. The content system is organized by the frame of nursing process, emphasizes human orientation, focuses on the health of nursing subjects, osmosis of human concern and care in a nursing evaluation to intervention and assessment, and shows the three-in-one service concept of body-psychology-society (12). It is widely believed that good clinical nursing adds strength to health, especially psychological health (13).

In conclusion, targeted nursing can significantly improve therapeutic compliance and clinical outcome of SLE patients and also reduce the incidence of complications and ameliorate quality of life. We also suggest that future studies should focus on targeted nursing for different complications that arise during SLE treatment and to evaluate the efficacy of targeted nursing and its clinical application. 


\section{References}

1. Jolly M and Utset TO: Can disease specific measures for systemic lupus erythematosus predict patients health related quality of life? Lupus 13: 924-926, 2004.

2. Huang X, Magder LS and Petri M: Predictors of incident seizure in systemic lupus erythematosus. J Rheumatol 43: $565-575,2016$.

3. Sheybani F, Naderi H, Mirfeizi Z and Erfani S: Sometimes there is more than one puzzle on the table: Pneumococcal bacteremia as a new systemic lupus erythematosus presentation. Case Rep Infect Dis 2015: 970289, 2015.

4. Shen B, Feng G, Tang W, Huang X, Yan H, He Y, Chen W, $\mathrm{Da} \mathrm{Z}$, Liu $\mathrm{H}$ and $\mathrm{Gu} \mathrm{Z}$ : The quality of life in Chinese patients with systemic lupus erythematosus is associated with disease activity and psychiatric disorders: A path analysis. Clin Exp Rheumatol 32: 101-107, 2014.

5. Waldheim E, Elkan AC, Pettersson S, van Vollenhoven R, Bergman S, Frostegård $\mathrm{J}$ and Welin Henriksson E: Health-related quality of life, fatigue and mood in patients with SLE and high levels of pain compared to controls and patients with low levels of pain. Lupus 22: 1118-1127, 2013.

6. Wheeler T: Systemic lupus erythematosis: The basics of nursing care. Br J Nurs 19: 249-253, 2010.
7. Hochberg MC: Updating the American College of Rheumatology revised criteria for the classification of systemic lupus erythematosus. Arthritis Rheum 40: 1725, 1997.

8. Chambers SA, Raine R, Rahman A and Isenberg D: Why do patients with systemic lupus erythematosus take or fail to take their prescribed medications? A qualitative study in a UK cohort. Rheumatology (Oxford) 48: 266-271, 2009.

9. Chen B, Tan W, Feng G, He Y, Liu J, Chen W, Huang X, Da Z, $\mathrm{Xu} \mathrm{X}$, Liu $\mathrm{H}$ and $\mathrm{Gu} \mathrm{Z}$ : The correlations of disease activity, socioeconomic status, quality of life, and depression/anxiety in Chinese patients with systemic lupus erythematosus. Clin Dev Immunol 2013: 270878, 2013.

10. Ferenkeh-Koroma A: Systemic lupus erythematosus: Nurse and patient education. Nurs Stand 26: 49-57, quiz 58, 2012.

11. Ito $H$, Matsuno $T$, Hirayama $T$, Tanino $H$ and Minami $A$ : Health-related quality of life in patients with systemic lupus erythematosus after medium to long-term follow-up of hip arthroplasty. Lupus 16: 318-323, 2007.

12. Zhang J, Wei W and Wang CM: Effects of psychological interventions for patients with systemic lupus erythematosus: A systematic review and meta-analysis. Lupus 21: 1077-1087, 2012.

13. Robinson M, Cook SS and Currie LM: Systemic lupus erythematosus: A genetic review for advanced practice nurses. J Am Acad Nurse Pract 23: 629-637, 2011. 